\title{
Data Mining in DHCP Security
}

\author{
D. Radha \\ M.O.P. Vaishnav \\ College for Women \\ Chennai
}

\author{
R. Jayaparvathy \\ M.O.P. Vaishnav \\ College for Women \\ Chennai
}

\author{
M. Shanmughi \\ M.O.P. Vaishnav \\ College for Women \\ Chennai
}

\author{
V. Jothilakshmi \\ M.O.P. Vaishnav \\ College for Women \\ Chennai
}

\begin{abstract}
A dynamic IP address is an IP address that is dynamically assigned to your computer by your Internet service provider (ISP). Once your computer or router is refreshed, your ISP dynamically assigns an IP address to your networking device using DHCP protocol. DHCP follows sequence in assigning an IP address to the host. If a particular host find its IP address it can easily know another host's ip address through random search. This paper concentrates on giving an insight about DHCP security.
\end{abstract}

\section{Keywords}

Data mining, data warehousing, dynamic IP address, network, DHCP, ping

\section{INTRODUCTION}

\subsection{Wire Shark}

Applying data mining in wire shark is to categorize the traffic flows based on packets moving from one place to another place. It is also used for running multimedia-rich, real and non-real time applications. This tool is designed for line sniffing data trunk (with prior permission) that is serving a network with tens of desktops, laptops, controllers and access points (AP's).

- Wire shark is the world's most popular network analyzer with over 50,000 downloads per month.

- It's available at a free of cost.

- Created by Gerald combs under the original name Ethereal, wire shark is maintained by a dedicated group of core developers.

- It is used in many industries and educational institutions.

- It is the continuation of a project that started in 1998.

\subsection{Some of the wire shark features}

- Deep inspection of hundreds of protocols, with more being added all the time.

- $\quad$ Live capture and offline analysis.

- Standard three-pane packet browser.

- Multi-platform:- Runs on windows, Linux, OS, Solaris, free BSD, net BSD and many others.

- Captured network data can be browsed via a GUI or via the TTY-mode T shark utility.

- The most powerful display filters in the industry.

- $\quad$ Rich VOIP analysis.

- Reads/writes many different capture file formats:
TCP dump (lib cap), PCAP NG, catapult DC T2000, CISCO secure IDS ip log, Microsoft network monitor, Network general sniffer (compressed and uncompressed), sniffer pro and net $\mathrm{x}$-ray, network instrument observer, net screen snoop Novel LAN analyzer, RADCAM WAN/LAN analyzer Shomiti / finiser surveyor, Tektronix $\mathrm{k} 12 \mathrm{xx}$, visual networks, visual uptime, wild packets ether peek, Token peek/ AIRO peek and many others.

- Captured files can be compressed with GZIP and also can be decompressed on the fly.

- $\quad$ Live data can be read from Ethernet, IEEE 802.11, PPP/HDLC, ATM, Bluetooth, USB, Token ring, frame relay, FDDI and others (depending upon your platform).

- Decryption support for many protocols, including IP sec, ISAKMP, Kerberos, SNMPv3, SSL/TLS, WEP and WPA/WPA2

- Coloring rules can be applied to the packet list for quick, intuitive analysis.

- Output can be exported to XML, POST SCRIPT, CSV or PLAIN TEXT.

\subsection{Functionality of wire shark}

- Wire shark has a graphical front end plus some integrated sorting and filtering options.

- Wire shark lets the user put network interface controllers that support promiscuous, so configured address and broadcast/multicast traffic.

- However, when capturing with a packet analyzer in promiscuous mode on a port on a network switch, not all traffic through the switch is necessarily sent to the port where the capture is done, so capturing in promiscuous mode is not necessarily sufficient to see all network traffic.

- Port mirroring or various network taps extend capture to any point on the network.

\subsection{Features}

- Data can be captured "from the wire" from a live network connection or read from a file of already captured packets.

Wire shark is a software that "understands" the structure (encapsulation) of different networking protocols. It can parse and display the fields, along with their meanings as specified by different networking protocols. Wire shark uses PCAP to capture packets, so it can only capture packets on the types of networks that PCAP supports. 
- $\quad$ PCAP - packet capture

In the field of computer network administration, PCAP consists of application programming interface (API) for capturing network traffic \}.

\subsection{Wire shark in data mining}

- There are numerous packet classification categories based on how packets are decoded and analyzed including the consideration of packet size, duration ,patterns of transmissions and bursts.

- Live data help the team leader/manager to check their co-workers whether their work going properly.

- Past data helps business peoples for business purposes and also for security purposes.

\subsection{Dynamic IP address}

Dynamic Host Configuration protocol, a protocol for assigning dynamic IP addresses to devices on a network. With dynamic addressing, a device can have a different IP address every time it connects to the network. In some systems, the device's IP address can even change while it is still connected.

\subsection{DORA}

When one installs a DHCP server into our network then DHCP server works on the basis of DORA process, first DHCP sends a hello message in to the network to discover the clients pc and when any client pc selects any IP from DHCP server provide that IP to client $\mathrm{pc}$ and both send acknowledgement to each other. This process is called DORA process.

\section{LITERATURE REVIEW}

\subsection{International journal of advanced technology}

Traffic engineering: Traffic engineering is the concept referring to a systematic process where traffic flows are arranged into "classified" groups for simplifying the transmission throughput networks and decreasing the change of congestions. It inherently capable of dealing with large volume of traffic through traffic aggregation. Traffic flow exhibit volatility some traffic flow specifications, such as: bandwidth and volume. Fluctuations and unsteadiness of the traffic reduce the efficiency of Traffic engineering in the process of data mining. Some reasons are link exhaustion and connection termination, bandwidth fluctuations, burst effect etc..,

\section{WIRE SHARK IN DATA MINING (PDF)}

\subsection{Wire shark in data mining and data warehousing}

Wire shark can be used for both data mining and data warehousing. It collects the net traffic is called data warehousing (i.e. Browser history).Depending upon the data one can need browser and filtering the data based upon it is called data mining. It has a filter option which is used for mining. It contains field name, relation, value and range. Another packet like filter option is finding packets. One can easily find packets once one can have captured some packets or have read in a previously saved capture file. Find packets can be found in edit menu. It contains display filter, hex value, string, up and down.
Go to first packet:

This helps us to find the first packet.

Go to last packet.

Data can be collected if the network is not secured else one can collect it using the port numbers.

\subsection{Time Display Format And Time References}

While capturing packets it getting time stamped. These will be saved to the capture file, so they will be available for later analysis.

\subsection{Some of the presentation formats are}

- Date and time of day

- $\quad$ Time and day

- Seconds since beginning of capture

- Seconds since previous of capture packet

- Seconds since displayed of capture packet

- Seconds since epoch

The available precisions are automatic, seconds, decisions, cent seconds, milliseconds, microseconds and nano seconds.

\section{PROPOSED APPROACH}

IP address of our PC can be found simply by 'ipconfig/all'. Through this one can find what ip address is used in our PC and by knowing another host ip address one can simply ping that ip address. If there is a proper connection then pinging process will be succeed. Eg.'ping 192.168.16.15'.Enable wire shark before the pinging process. Wire shark starts the capturing process. When pinging process get succeeded one can get the mac address by applying filters. Wire shark filter the mac addresses.

One can virtually test this process by using GNS and Wire shark by connecting two PC through modem or switch and give configuration wherever necessary. Ping and capture the process as wire shark can work on both real and non real time.

In this approach one can find the MAC address for the host which one can know already by pinging process.

\subsection{System Model}

DHCP allocates IP address dynamically to the pc's or mobiles or any wireless or wired networks.. But this is not a permanent address like MAC address or ip address used in static network. If one can watched closely the ip address follows a sequence like the 192.168.1.11, 192.168.1.12, 192.168.1.13 or192.168.1.2, 192.168.1.4, 192.168.1.5 or 192.168.1.3, 192.168.1.6, 192.168.1.9. Dynamic networks are mostly used in local places like home, small shops. Static network is used in factories or colleges cause it is a dedicated network, it will be of more worth only when use the internet for which one paid or else it will be in vain and it is not cost effective.

Dynamic network is not a dedicated network but it will supply network based upon our budget. As the DHCP allocates the IP address automatically while rebooting and there is less security. Here data mining is applied using Wire shark.

Enable the wire shark before pinging. First find our ip address then ping the ip address randomly like in a sequential order or with the difference of $2,3,4,5, \ldots . .10$ etc... If our wireless 
network can connect 5 systems then one can find at least 3 out of 5 devices MAC address.
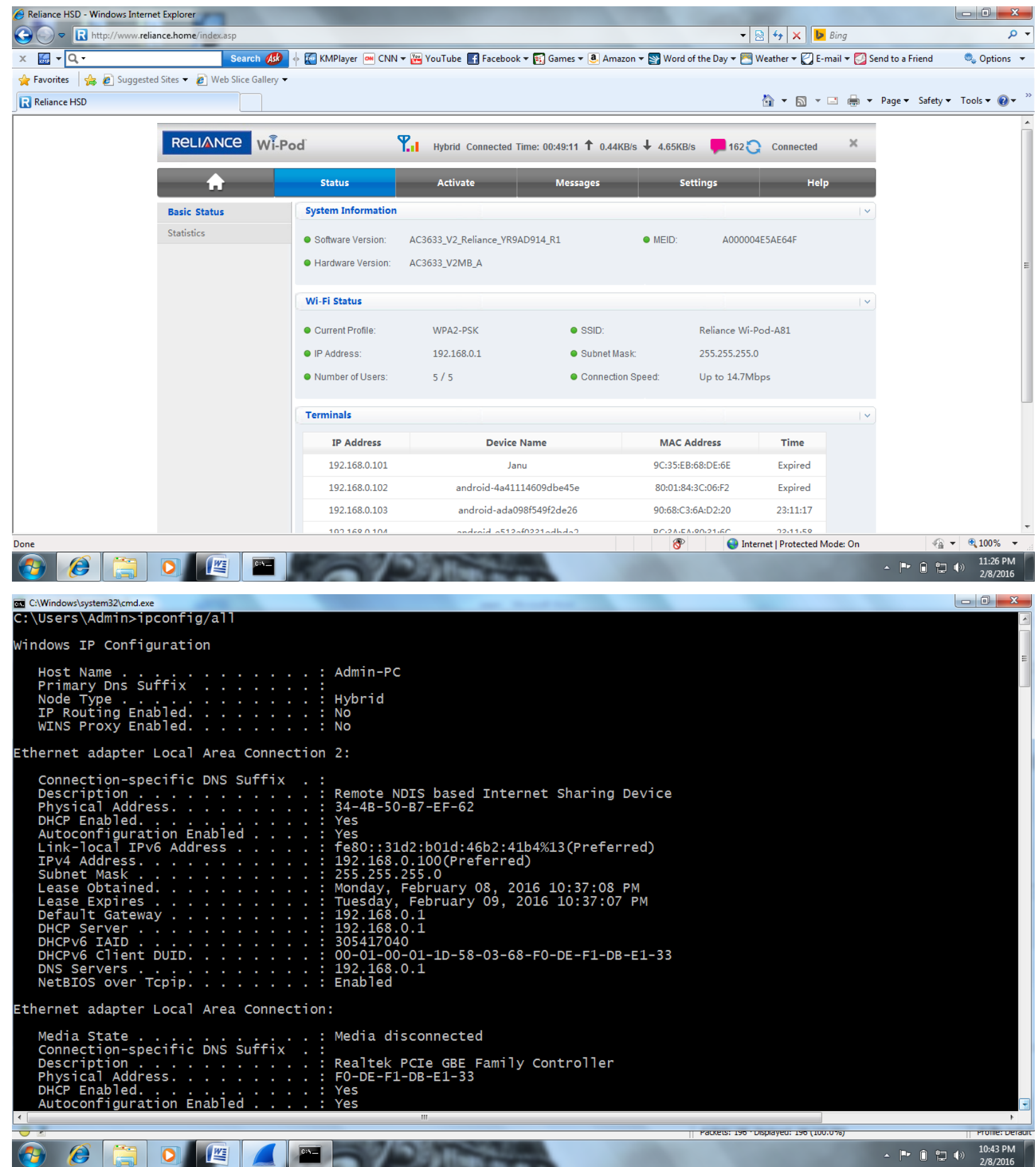

First I'm knowing my systems IP address. From this I'm applying a random approach like dhcp first allocates IP address where it is directly connected. This network can support 5 devices and one PC or laptop. So from this I can guess how devices I can connect. 


\subsection{Applying in Reliance}
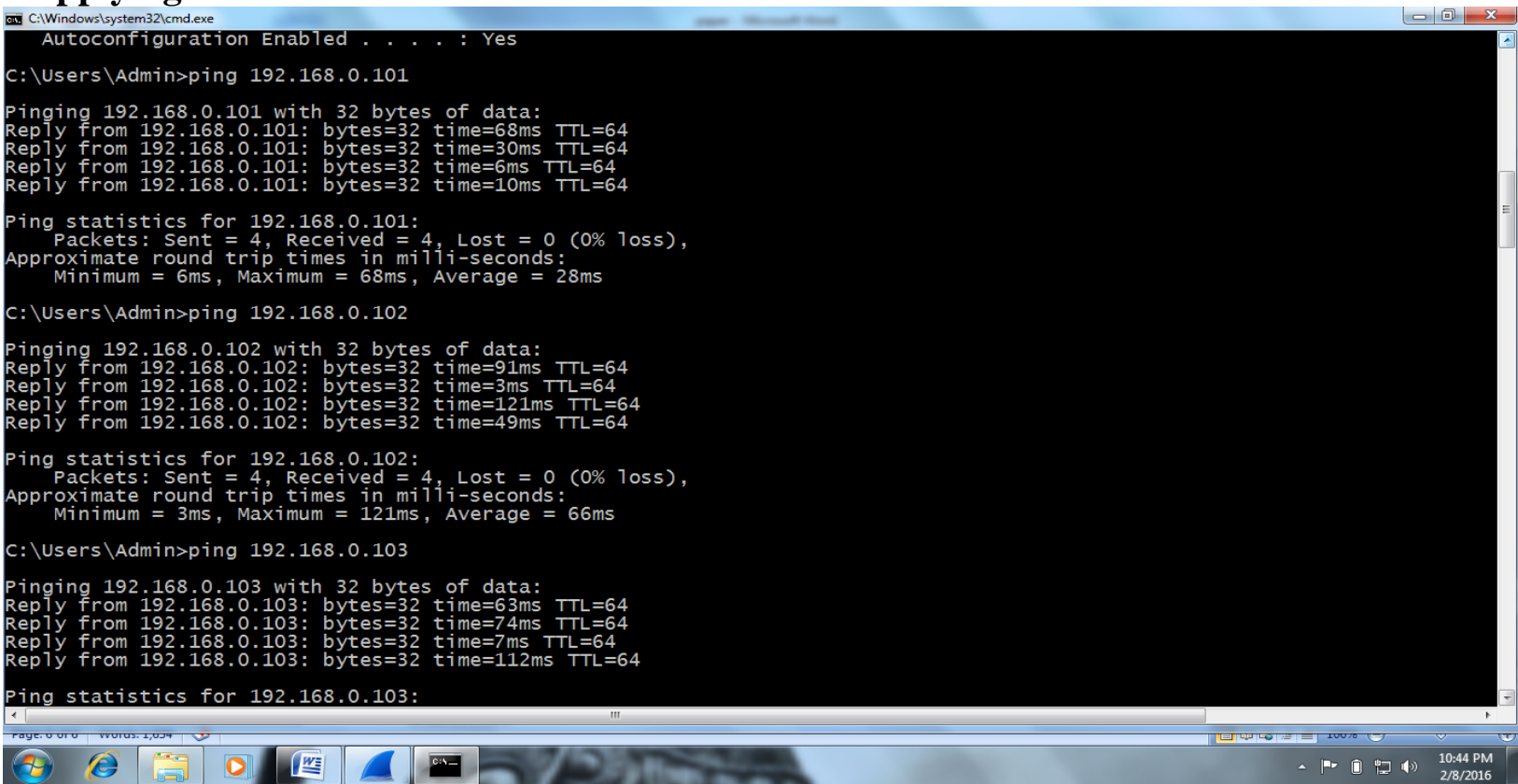

My system is the first device so it automatically allocates first ip address to my system. So first I am applying sequential order and it works so I started pinging 5 ip addresses randomly. I not checked those devices whether it really have that ip address or not.

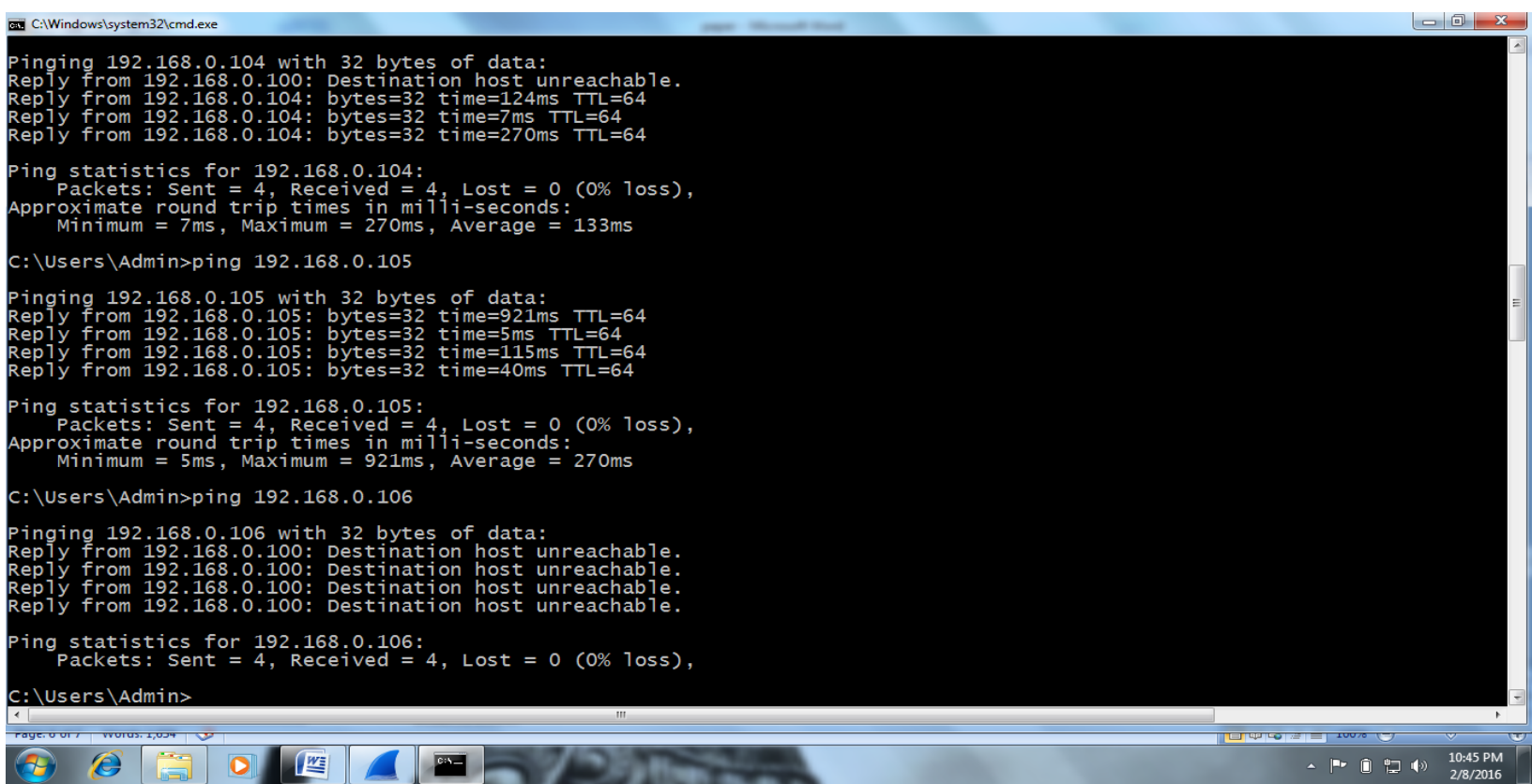

In the above picture after successful ping of 5 ip address and I'm trying to ping the sixth device where it not exist. So I'm getting a message like destination host unreachable. 


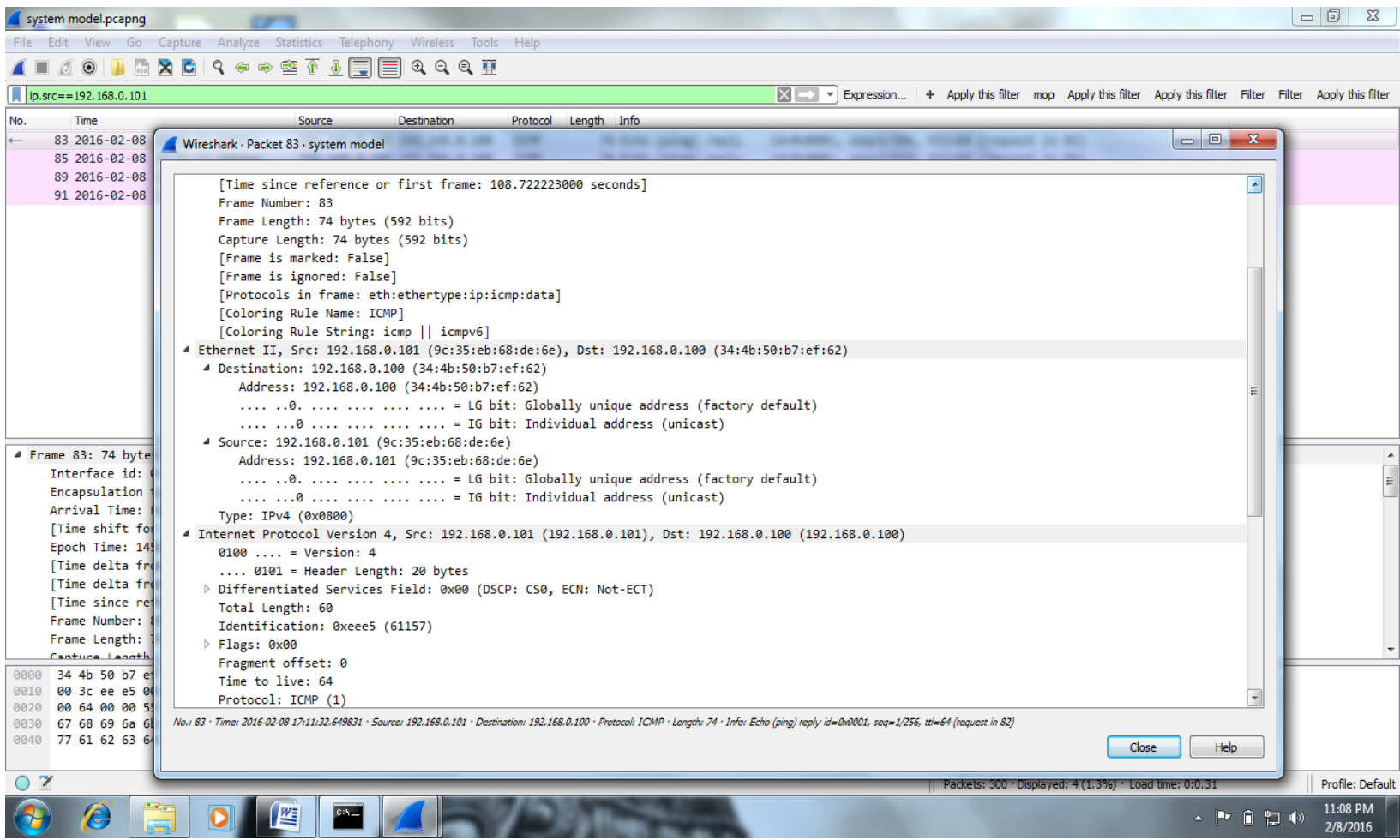

The above picture is the result of the filtered result from wire shark.

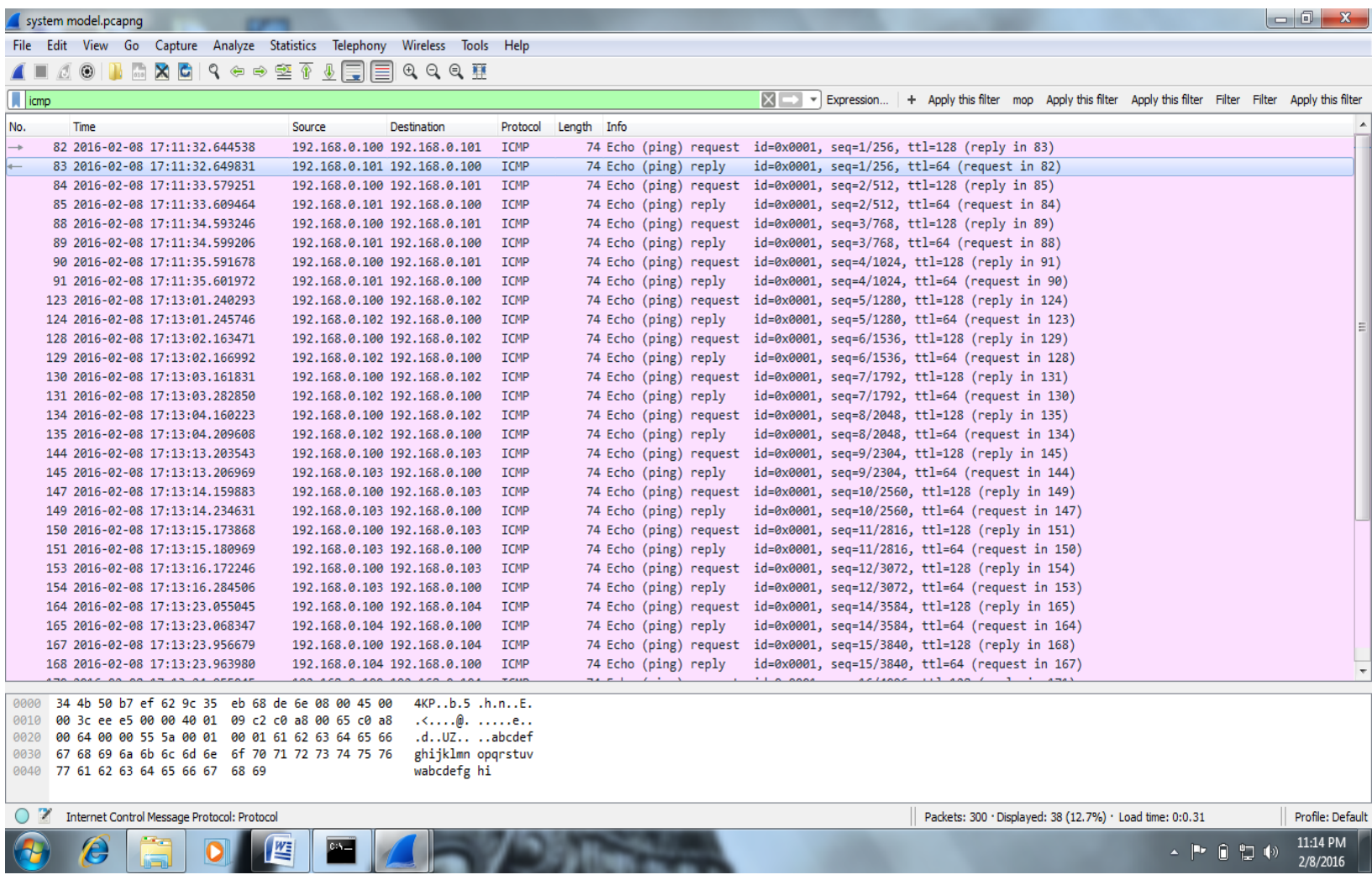




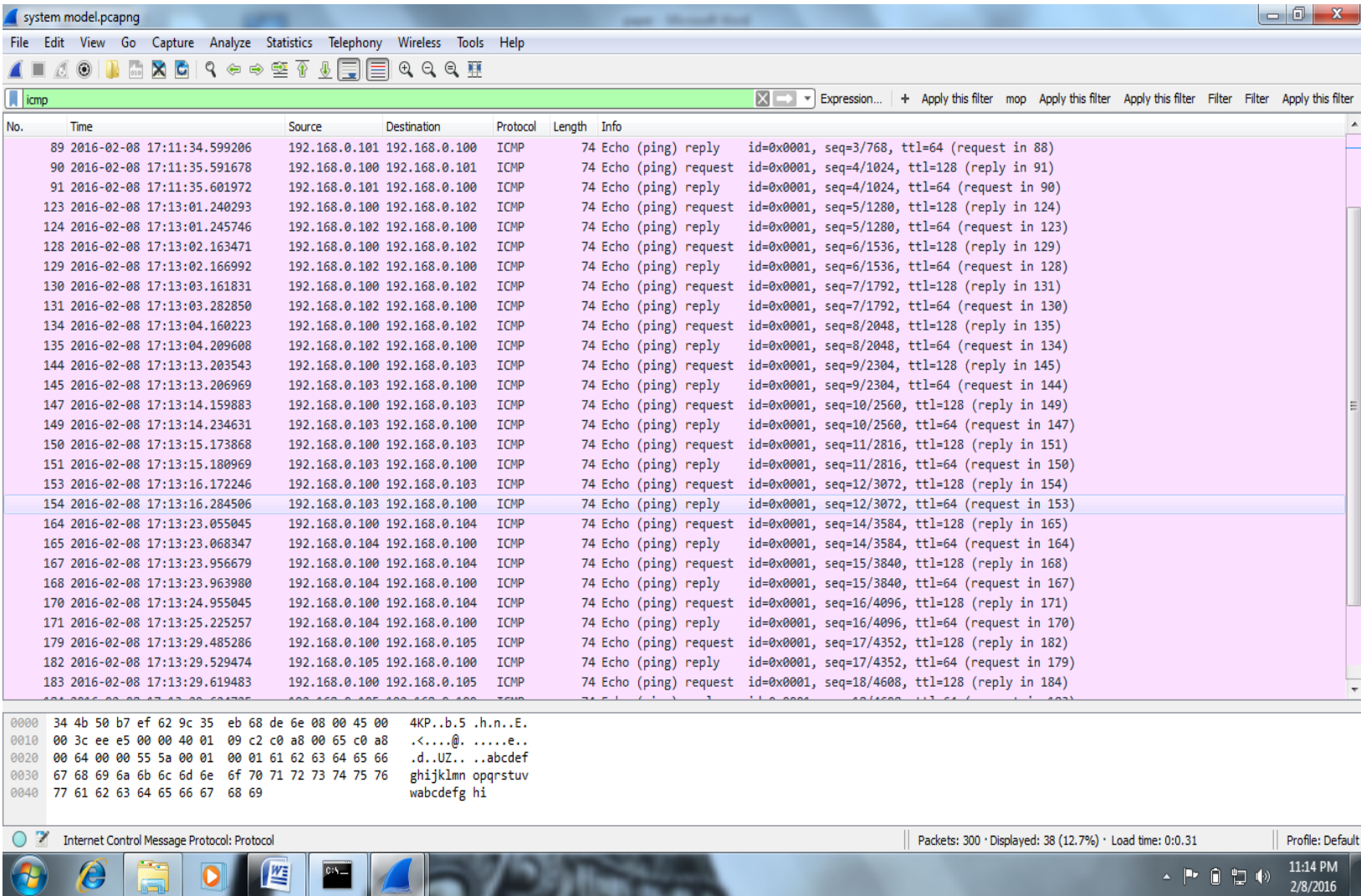

4 system model.pcapng

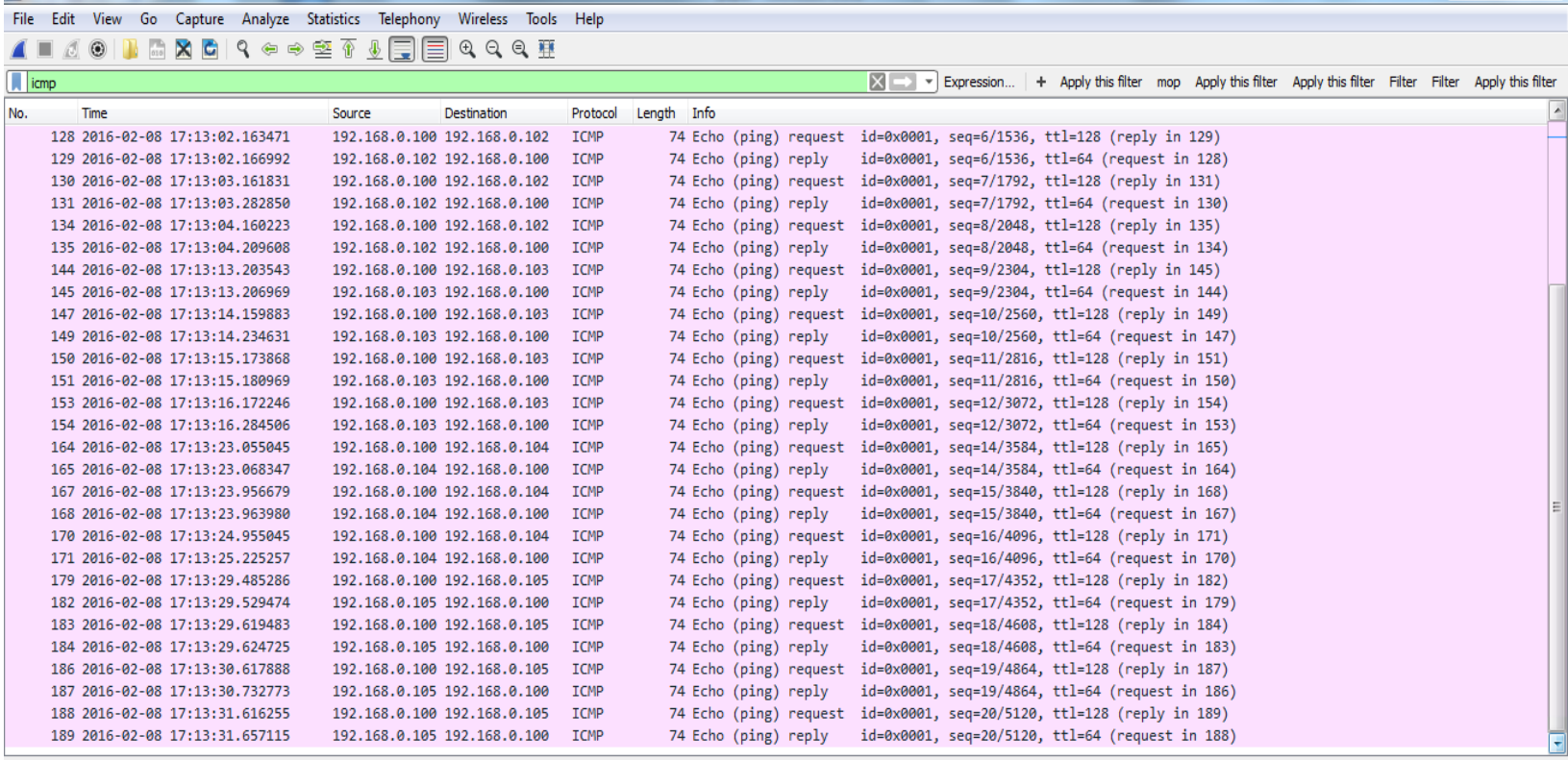

\begin{tabular}{|c|c|c|c|}
\hline & $344 b 50$ b7 ef 62 ac 35 & eb 68 de $6 e \quad 08004500$ & 4KP..b.5 h.h.n..E. \\
\hline 0010 & $00 \quad 3 c$ ee e5 $00 \quad 00 \quad 4001$ & $09 \mathrm{c2} c 0$ a8 $00 \quad 65 \mathrm{c \theta}$ a8 & $<\ldots \ldots @$. \\
\hline 0020 & 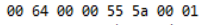 & $\begin{array}{llllllll}00 & 01 & 62 & 63 & 64 & 65 & 66\end{array}$ & .d..uz.. ..abcdef \\
\hline 0030 & $6768 \quad 69 \quad 6 \mathrm{a} \quad 6 \mathrm{~b} \quad 6 \mathrm{c} 6 \mathrm{~d} 6 \mathrm{e}$ & 6f $70 \quad 7172 \quad 73 \quad 74 \quad 75 \quad 76$ & ghijklmn opqrstuv \\
\hline 0840 & $\begin{array}{llllllll}77 & 61 & 63 & 64 & 65 & 66 & 67\end{array}$ & $68 \quad 69$ & wabcdefg hi \\
\hline
\end{tabular}

\begin{tabular}{|l} 
(2) Internet Contol Message Protocol: Protocol \\
\hline (2)
\end{tabular}

The filtered process can be get by giving the command 'ICMP'. This will be helpful to get the information of both source and destination.

First think of how many devices it can accept. Then how many devices already got connected. Then one have to apply guess based upon it. For example my device is connected as fifth and already some devices are working means then I'll be guessing reversely. If three devices working means both forward and reverse approach. 


\subsection{Applying in BSNL}
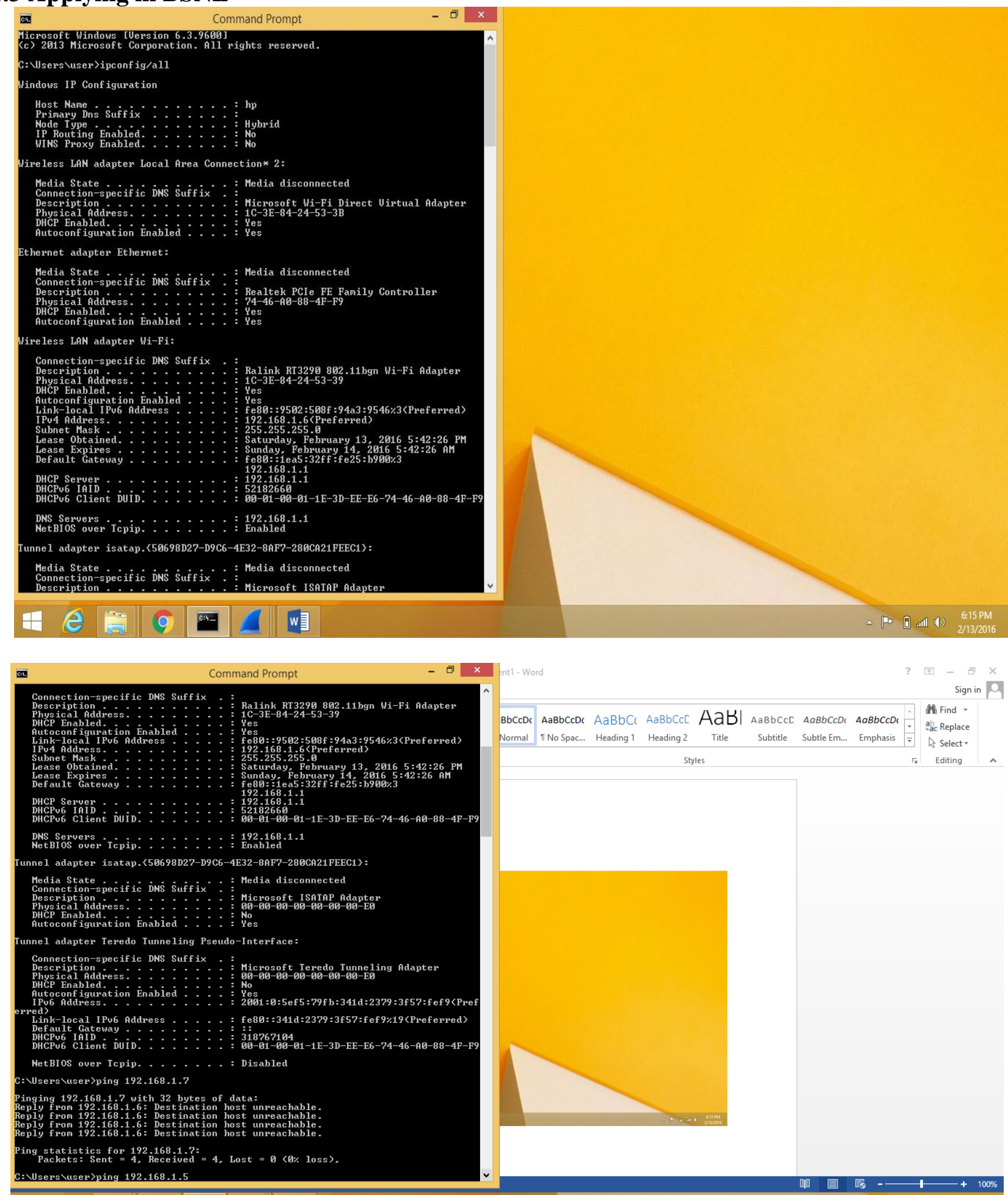

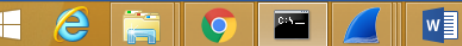




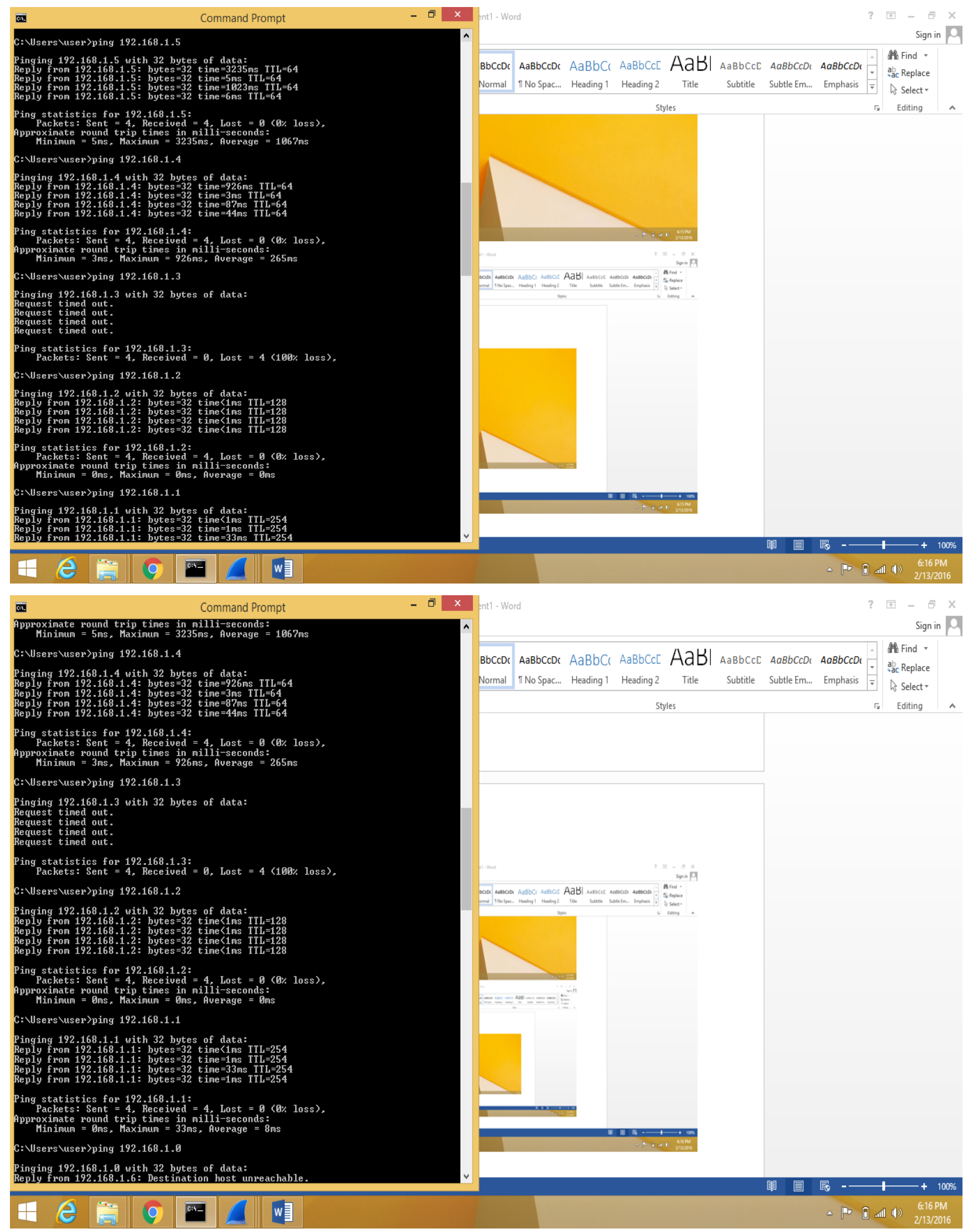




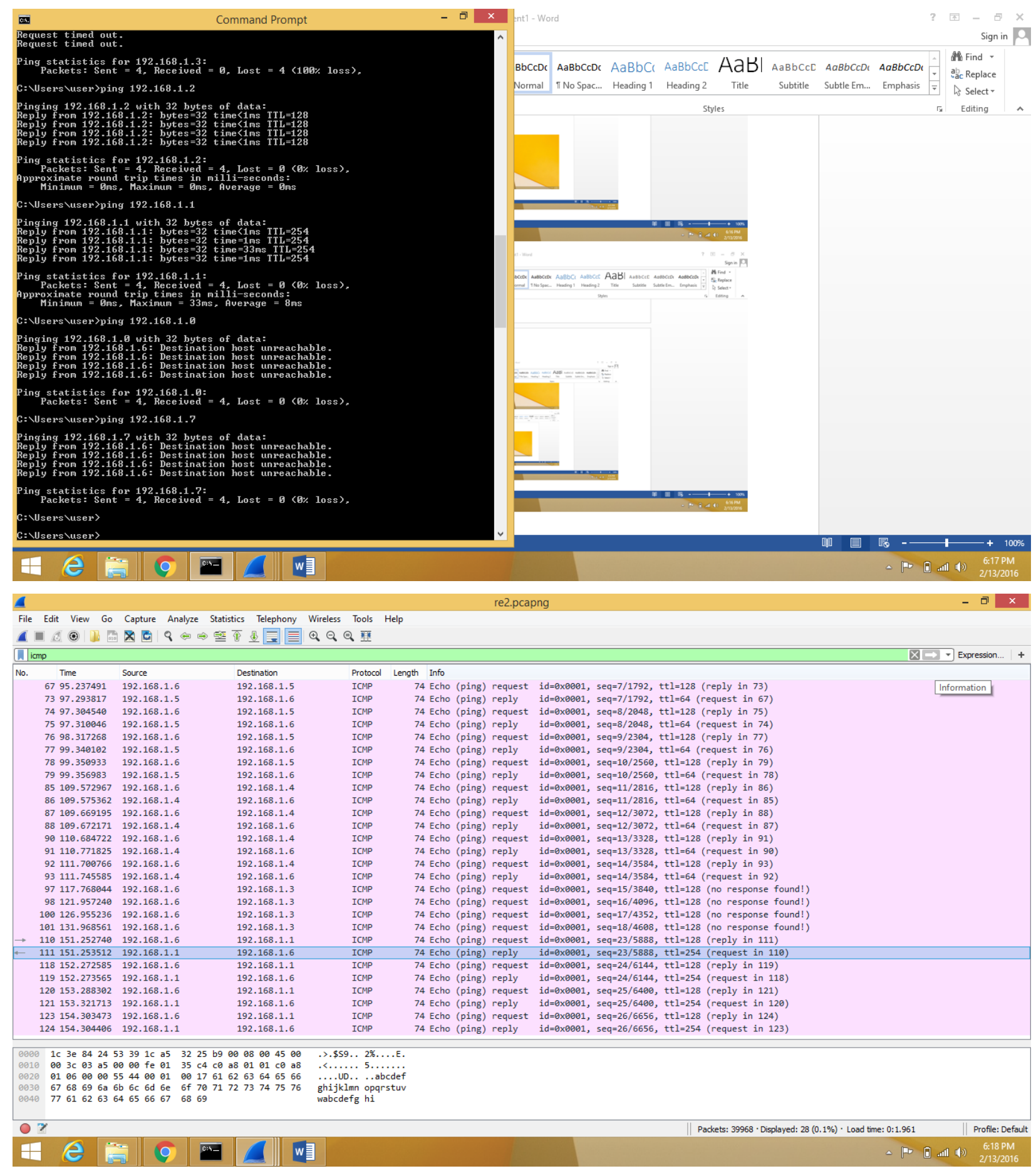




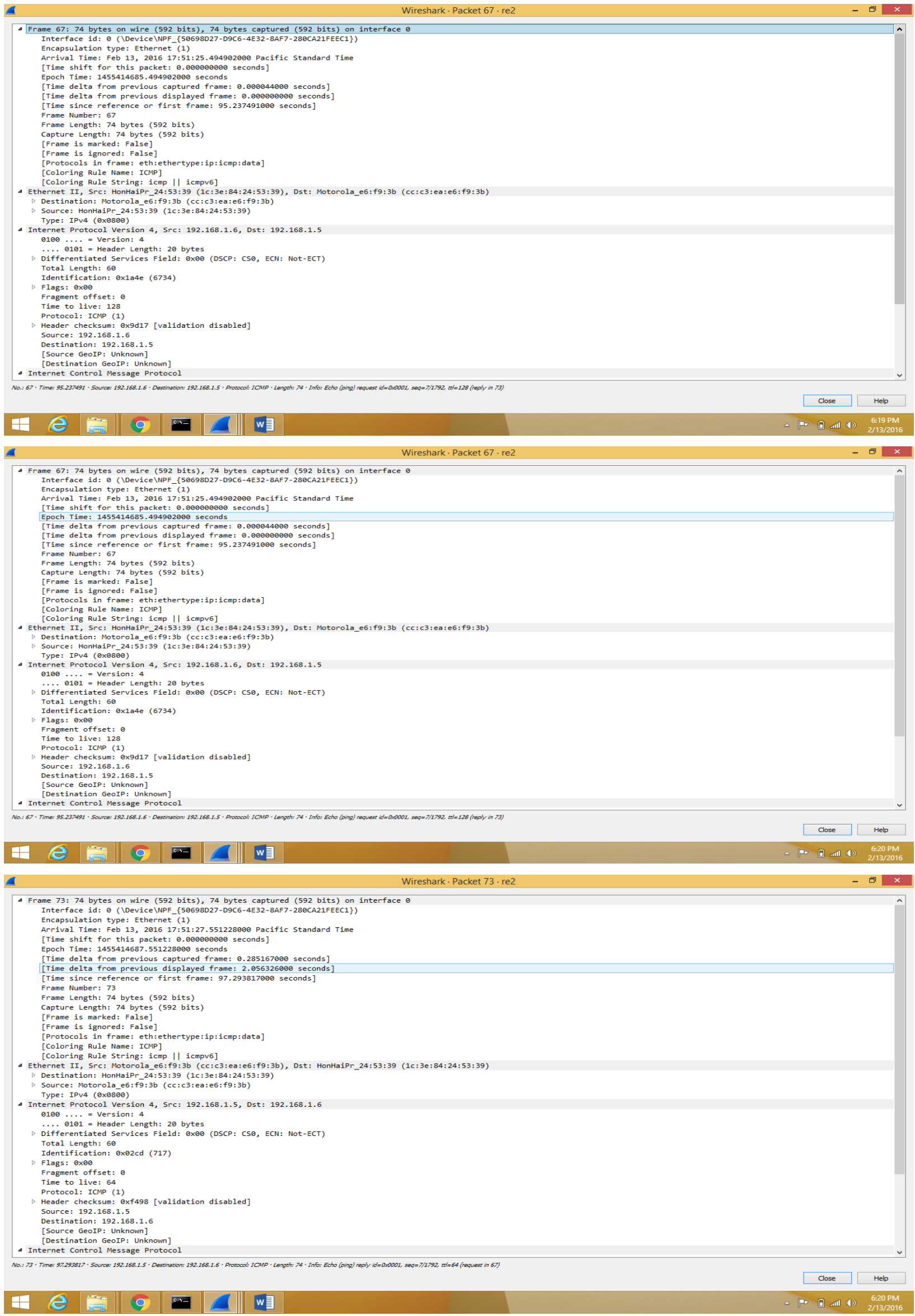




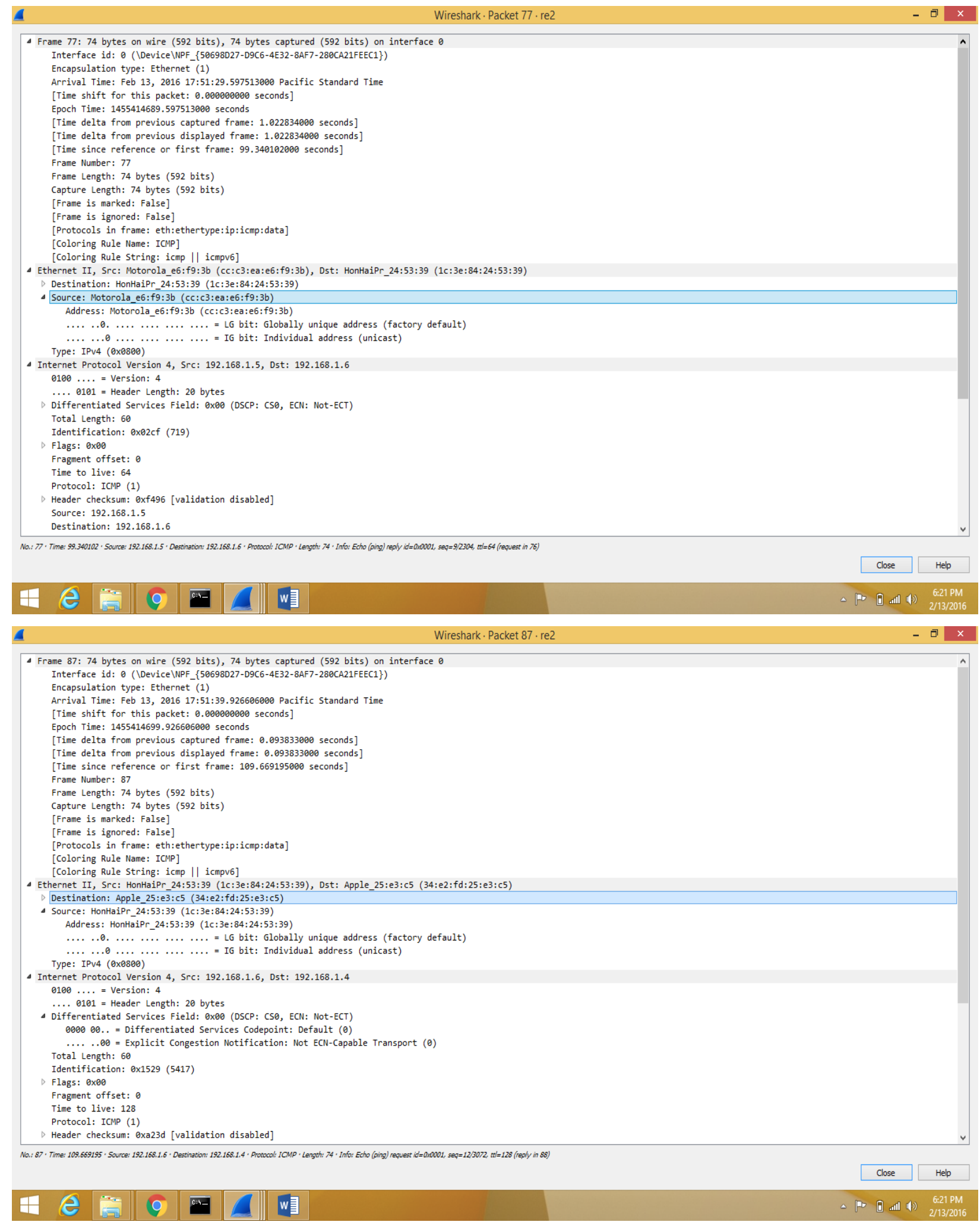




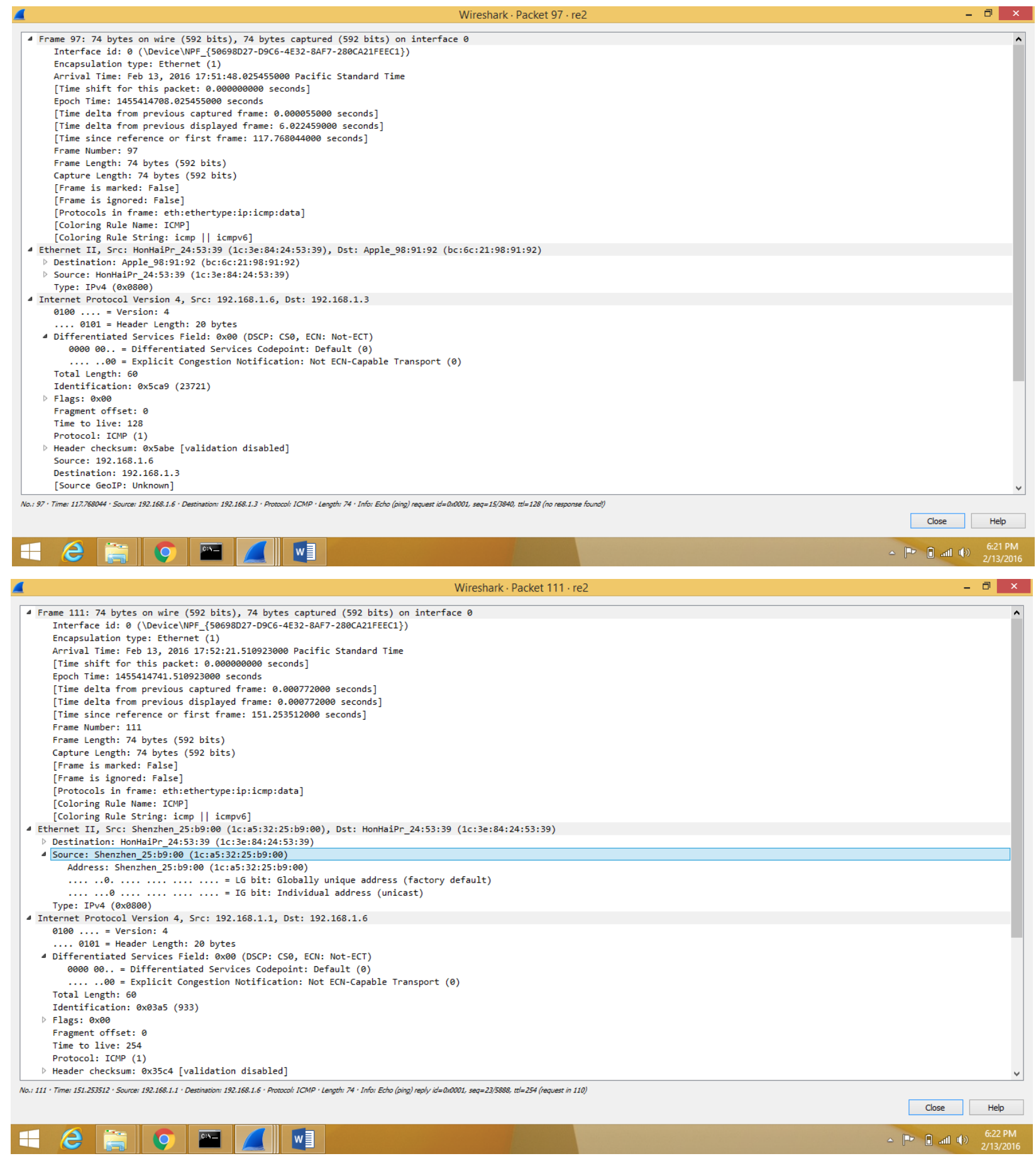

\section{CONCLUSION}

Data mining has been discussed in this paper. IP address plays a vital role in today's network. Hence it also needs protection like http turned into https for security needed websites or it needs some encryption for the PC's which has some important files. This random approach can be done by anyone who has little knowledge about ip address hence protection is needed for PC's. Wire shark tool is an ethical tool. An ethical tool itself finds mac address so easily means naturally one has to worry about the security of IP address.

\section{REFERENCES}

[1] http://www.wireshark.org//

[2] http://www.packets.com//

[3] http://www.network.com//

[4] http://www.linkage.rockfeller.edu/wli/zipf/org// 